\title{
Ero straniero. Qualche statistica, alcuni materiali, qualche spunto di riflessione
}

\author{
I was a stranger. Some reflection
}

\author{
Agata C. Amato Mangiameli \\ Universitá degli Studi di Roma «Tor Vergata» (Italia) \\ amato@juris.uniromaz.it
}

\begin{abstract}
Riassunto: L'Europa è al bivio: potrebbe essere tentata di fronte alle masse di stranieri di chiudere la porta (in terraferma di erigere muri, di stendere fili spinati, in mareaperto di respingere e violare la legge del mare), ma questa porta verrebbe inevitabilmente abbattuta; oppure, di tenerla aperta, governando il fenomeno con politiche di accoglienza e di cooperazione, creando nuove opportunità e diritti per i diversi profughi (di guerra, politici, economici, ambientali). L'immigrazione sollecita i temi della pace, dei diritti, dell'ambiente, ed è innanzitutto storia individuale, la storia di chi chiede ospitalità e nonviolenza.
\end{abstract}

Parole chiave: stranieri; immigrazione; pluralismo culturale; cittadinanza.

\begin{abstract}
Europe is at a crossroad: in front of masses of foreigners it could be tempted to close the door. It would mean, on the earth, to erect walls or stretch spiny yarns. On open sea, it would mean to repudiate and violatie the law of the sea. As a matter of fact, this door would inevitably be demolished. On the other hand, Europe could decide to keep the door open and try to govern this phenomenon adopting welcome and cooperation policies, creating new opportunities and rights for different types of refugees (political, economic, environmental). All of the mentioned suggests reflecting on peace, rights and the environment. Immigration is first of all and foremost an individual story, the story of those who seek hospitality and non-violence.
\end{abstract}

Keywords: strangers; immigration; pluralism; citizenship.

1. Ero straniero - L'umanità che fa bene, è il nome della campagna di raccolta firme sulla proposta di legge di iniziativa popolare volta ad introdurre una nuova politica sulle migrazioni, e cioè: $i$ ) il superamento della legge Bossi-Fini; ii) la soluzione circa le possibili situazioni di irregolarità; iii) la ricerca di integrazione grazie al combinarsi di accoglienza-lavoro-inclusione ${ }^{1}$.

Più in particolare, gli articoli contenuti nella proposta di legge presentata alla Camera dei Deputati (n. 4712 del 27 ottobre 2017) prevedono: a) l'intro-

1 Lanciata il 12 aprile del 2017 la campagna mirava a raccogliere, nell'arco di sei mesi, le 50.000 firme richieste per poter sottoporre all'attenzione del Parlamento la legge di iniziativa popolare dal titolo «Nuove norme per la promozione del regolare permesso di soggiorno e dell'inclusione sociale e lavorativa di cittadini stranieri non comunitari». Conclusasi il 27 ottobre 2017, la campagna ha avuto un successo anche superiore alle attese: $150 \mathrm{i}$ comuni che hanno aderito, $4.000 \mathrm{i}$ banchetti allestiti in tutto il territorio nazionale, 90.000 le firme raccolte. 
duzione di un permesso di soggiorno temporaneo di un anno per la ricerca di occupazione, affidando l'intermediazione tra datori di lavoro italiani e lavoratori stranieri alle agenzie preposte o a onlus iscritte in apposito registro; $b$ ) la reintroduzione dello sponsor, già contemplato nella legge Turco-Napolitano, e cioè di un cittadino italiano che garantisce l'ingresso dello straniero; c) la regolarizzazione su base individuale degli stranieri già integrati in Italia, nuovi standard per riconoscere le qualifiche professionali e misure di inclusione attraverso il lavoro dei richiedenti asilo; $d$ ) il godimento dei diritti previdenziali e di sicurezza sociale una volta tornati nel Paese d'origine con la possibilità di ritirare l' $80 \%$ dei contributi versati; e) l'uguaglianza nelle prestazioni di sicurezza sociale e maggiori garanzie per un reale diritto alla salute dei cittadini stranieri; il voto amministrativo e l'abolizione del reato di clandestinità ${ }^{2}$.

Il fronte dei promotori è stato decisamente ampio. Fra questi: i Radicali Italiani, la Fondazione Casa della Carità «Angelo Abriani», l'Acli, l'Arci, l'Asgi, il Centro Astalli, A buon diritto, Cnca, Cild. Liniziativa, inoltre, ha potuto contare sull'adesione e sul supporto di un cospicuo numero di organizzazioni (la Fondazione Migrantes, la Caritas italiana, la Comunità di Sant'Egidio, la Cgil, Altromercato, la Federazione Chiese Evangeliche Italiane, Emergency) e di personalità, che, in più occasioni, hanno espresso il proprio sostegno e il proprio plauso alla legge di iniziativa popolare volta a cambiare le politiche sull'immigrazione. D'altra parte, l'adozione di un governo dei flussi migratori - che sia capace di tramutare il fenomeno in un'opportunità per il Paese - e il necessario contrasto alla narrazione mediatica (spesso poco veritiera e allarmista) richiedono l'intervento di competenze di vario genere ed invitano ad un confronto e ad un dialogo fra le diverse sensibilità presenti nella società civile.

1.1. Contro i tanti luoghi comuni e contro le molte informazioni dubbie, il Piccolo prontuario per un racconto (finalmente) veritiero sull'immigrazione ${ }^{3}$ rende evidente quanto siano errate le affermazioni e le convinzioni del tipo: «sia-

2 Il testo integrale della proposta è disponibile sul sito della Camera dei Deputati al seguente indirizzo <http://www.camera.it/_dati/leg17/lavori/stampati/pdf/17PDL0056430.pdf> (22 gennaio 2018).

3 Il testo del Prontuario è contenuto in Sconfiggere la grande bugia e cambiare il racconto sull'immigrazione. Tutto quello che sai sugli immigrati è falso! Ed è disponibile on-line al seguente indirizzo: <http://www.radicali.it/bugie-sull-immigrazione/> (sito visitato il 22 gennaio 2018). 
mo di fronte a un'invasione ${ }^{4}$, $^{\prime}$ non c'è lavoro neanche per gli italiani, non possiamo accoglierli» ${ }^{5}$, «questi ci rubano il lavoro e ci tolgono risorse per il welfare ${ }^{6}, \ll$ rifugiati sono troppi, non c'è abbastanza spazio in Europa $»^{7}, \ll l i$ ospitiamo negli alberghi e diamo loro 35 euro al giorno per non fare niente ${ }^{8}$, $\ll i$ terroristi islamici stanno sfruttando i flussi migratori per fare attentati e conquistare l'Europa» ${ }^{9}$.

Al di là dei contenuti del Prontuario, è interessante sottolineare che a seguito della comparazione dei dati relativi al numero dei migranti sbarcati negli anni 2016, 2017 e 2018, il Ministero dell'Interno evidenzia che, nel 2016, sono arrivate 181.436 persone, nel 2017, 119.369, e che, nell'arco del primo mese del 2018 (dal 1 al 24 gennaio), sono sbarcate 2.730 (contro le 2.486 del

4 In realtà, nell'Unione Europea la quota di immigrati residenti rispetto all'intera popolazione sarebbe di circa il 7\%. Nei Paesi europei tale quota varierebbe sensibilmente (ad esempio: il $10 \%$ in Spagna, il 9\% in Germania, l'8\% nel Regno Unito e in Italia, il 7\% in Francia)

5 Si legge nel Prontuario, «per mantenere sostanzialmente inalterata la popolazione italiana dei 15-64enni nel prossimo decennio, visto che tra il 2015 e il 2025 gli italiani diminuiranno di 1,8 milioni, è invece necessario un aumento degli immigrati di circa 1,6 milioni di persone: si tratta di un fabbisogno indispensabile per compensare la riduzione della popolazione italiana in età lavorativa».

6 A tal proposito si osserva innanzitutto che agli immigrati sono riservati solo i lavori non qualificati, in gran parte rifiutati dagli italiani. In altri termini, «gli stranieri non riducono l'occupazione degli italiani, ma occupano progressivamente le posizioni meno qualificate abbandonate dagli autoctoni, soprattutto nei servizi alla persona, nelle costruzioni e in agricoltura: settori in cui il lavoro è prevalentemente manuale, più pesante, con remunerazioni modeste e con contratti non stabili». Si osserva inoltre che «i costi complessivi dell'immigrazione, tra welfare e settore della sicurezza, sono inferiori al $2 \%$ della spesa pubblica».

7 Per il vero è sufficiente considerare che «dei 16 milioni complessivi solo 1,3 milioni sono ospitati nei 28 Paesi dell'Unione europea (8,3\%), tra cui l'Italia (118mila, pari allo 0,7\%). I Paesi che ospitano il maggior numero di rifugiati nel 2015 sono la Turchia (2,5 milioni), il Pakistan (1,6 milioni), il Libano (1,1 milioni) e la Giordania (664 mila)».

8 Scelta che, in realtà, si rivela necessaria in quanto i centri di accoglienza straordinaria sono strutture temporanee e in considerazione di ciò le prefetture, insieme alle Regioni e agli enti locali, cercano ulteriori posti di accoglienza nei singoli territori regionali, coinvolgendo anche le strutture alberghiere. Per quanto riguarda poi i 35 euro al giorno, si tratta del costo medio per l'accoglienza di un richiedente asilo o rifugiato, copre quindi i diversi costi di gestione, compreso il cosiddetto pocket money (2,5 euro circa) dato al migrante.

$9 \mathrm{Al}$ riguardo si obietta: «limitando l'osservazione al terrorismo islamista, i primi 5 Paesi con la maggiore quota di morti sono l'Afghanistan (25\%), l'Iraq (24\%), la Nigeria (23\%), la Siria $(12 \%)$, il Niger (4\%) e la Somalia (3\%). Le vittime dell'Europa occidentale rappresentano una quota residuale, inferiore all'1\%. L'Italia è terra d'immigrazione con molti cristiani ortodossi: oltre 2 milioni tra ucraini, romeni, moldavi e altre nazionalità. Seguono circa 1 milione e 700mila persone di religione musulmana (compresi gli irregolari e minori), meno di un terzo del totale degli oltre 5 milioni di stranieri in Italia. In Europa solo il 5,8 per cento della popolazione è di religione islamica». 
gennaio 2016 e le 2.790 del 2017). Dati che sembrano dimostrare una seppur lenta riduzione degli afflussi ${ }^{10}$.

Inoltre, con specifico riguardo alle domande d'asilo (che vengono esaminate da 20 commissioni territoriali deputate alla verifica dei presupposti), è interessante sottolineare che, nel corso del 2016, sono state prese 91.102 decisioni (a fronte di 123.600 domande fatte), di cui 4.808 riconoscimenti dello status di rifugiato (5\%), 2.873 riconoscimenti della protezione sussidiaria (14\%), 18.979 proposte di rilascio del permesso di soggiorno per motivi umanitari (21\%), 51.170 dinieghi (56\%). Diversamente, nell'arco di tutto il $2017^{11}$ sono state prese 77.553 decisioni, di cui 6.578 riconoscimenti dello status di rifugiato (8\%), 5.680 riconoscimenti della protezione sussidiaria (7\%), 18.951 proposte di rilascio del permesso di soggiorno per motivi umanitari (24\%), 46.186 dinieghi (59\%).

I dati relativi a minori stranieri non accompagnati sbarcati riportano un particolare incremento rispetto al 2015 (12.360), visto che nell'anno 2016 sono stati 25.846 e che, nel corso del 2017 , sono stati $18.508^{12}$. A tutto ciò va aggiunto il fenomeno - importante per i diversi risvolti, oltre che per l'evidente gravità - dei minori non accompagnati irreperibili.

Questi dati - come pure ogni altro - devono essere valutati in combinazione con quanto previsto dal World Population Prospects secondo cui nel 2050 la popolazione mondiale aumenterà dagli attuali 7,3 a 9,7 miliardi, oltre la metà di tale crescita interesserà l'Africa, dove la popolazione raddoppierà e raggiungerà i 2,5 miliardi, di cui 450 milioni saranno solo nigeriani.

1.2. Il Mediterraneo, fonte di ispirazione per Paul Valery ${ }^{13}$, è diventato ormai da tempo un mare diviso e lacerato. Piuttosto che fabbricare civiltà,

10 Cfr. <http://www.interno.gov.it/it/sala-stampa/dati-e-statistiche/sbarchi-e-accoglienza-dei-migranti-tutti-i-dati> (visitato il 24 gennaio 2018). Per un commento e un approfondimento ulteriore, si veda Sarti, S., L'Italia e il popolo dei rifugiati, in Amato MangIameli (a cura di), Immigrazione integrazione marginalizzazione, Giappichelli, Torino (in corso di stampa).

11 I dati relativi al 2017, suddivisi per mese, sono disponibili al seguente link <http://www.libertaciviliimmigrazione. dlci. interno. gov. it/ it/ documentazione/statistica/i-numeri-dellasilo $>$ (visitato il 4 gennaio 2018).

12 Come risulta dal più recente Report mensile stranieri non accompagnati in Italia aggiornato al 30 novembre 2017 <http://www.lavoro.gov.it/temi-e-priorita/immigrazione/focus-on/minori-stranieri/Documents/Report-MSNA-mese-novembre-30112017.pdf> (visitato il 24 gennaio 2018).

13 Inspirations Méditerranéennes (1940), trad. it., Ispirazioni mediterranee, Mesogea, Messina, 2011. 
coltivare e valorizzare la cultura del limes, dei molti dèi e delle tante lingue, è il centro delle principali tensioni geopolitiche. Il mare nostrum si è trasformato in un mare nel quale Mamadou va a morire ${ }^{14}$.

Si pensi alle vittime in attesa del proprio turno per salire sopra imbarcazioni improvvisate, alle tante donne vittime di violenza prima della partenza, ai morti per naufragio. Alcune date significative: il 3 ottobre 2013, l'11 febbraio 2015, il 13 aprile 2015, tutte date in cui si sono verificati naufragi durante i quali hanno perso la vita centinaia e centinaia di persone, alle quali si aggiunge quella del 18 aprile 2015, data in cui si è registrato il naufragio più grave $^{15}$. Di quest'ultimo sono stati recuperati circa 700 corpi in un'operazione che in sé ha destato parecchie critiche, anche sulla base dell'argomentazione che la sepoltura in mare nelle sue diverse forme ha una sua tradizione e che non esisterebbe alcun principio etico universale a detta del quale i corpi dei migranti giacenti in fondo al mare andrebbero recuperati e seppelliti. In realtà, è agevole osservare che una cosa è «scegliere, per un defunto, il mare e non la terra come luogo della 'sepoltura', a maggior ragione se la scelta è dei familiari o di un qualche prossimo (il comandante della nave), che decide che si possono o si devono compiere le esequie attraverso questa forma; altro è, a motivo delle circostanze della morte, non aver potuto scegliere, né assistere a una 'sepoltura' che tale non è» ${ }^{16}$.

2. L'Europa è al bivio: potrebbe essere tentata di fronte alle masse di stranieri di chiudere la porta (in terraferma di erigere muri, di stendere fili spinati, in mare aperto di respingere e violare la legge del mare), ma questa porta verrebbe inevitabilmente abbattuta; oppure, di tenerla aperta, governando il fenomeno con politiche di accoglienza e di cooperazione, creando nuove opportunità e diritti per i diversi profughi (di guerra, politici, economici, ambientali). L'immigrazione sollecita i temi della pace, dei diritti, dell'ambiente, ed è innanzitutto storia individuale, la storia di chi chiede ospitalità e non-violenza.

14 Del Grande, G., Mamadou va a morire. La strage dei clandestini nel Mediterraneo, Infinito, Roma, 2008.

15 Piscitelli, V., L'identificazione delle vittime dei naufragi nel Mediterraneo: la sfida raccolta dell'Ufficio del Commissario straordinario del Governo per le persone scomparse, in AMATO MANGIAMELI, A.C. (a cura di), Immigrazione marginalizzazione integrazione, cit.

16 Mocellin, G., «Seppellire i morti», in Il regno - Attualità, 12 (2016), p. 335. 
Ero straniero, espressione tanto chiara, quanto antica. «Ho avuto fame e mi avete dato da mangiare, ho avuto sete e mi avete dato da bere; ero forestiero e mi avete ospitato, nudo e mi avete vestito, malato e mi avete visitato, carcerato e siete venuti a trovarmi». Solo sei azioni concrete, soltanto sei opere di misericordia corporale sono contenute nel Vangelo di Matteo, mentre la settima - quella di seppellire i morti quale ultimo gesto di amore e rispetto verso il defunto - è stata successivamente aggiunta dalla tradizione della Chiesa. Eppure tanto basta per trasformare il mondo in cui viviamo nella Terra promessa, o al contrario, se disattese («ho avuto fame e non mi avete dato da mangiare; bo avuto sete e non mi avete dato da bere; ero forestiero e non mi avete ospitato, nudo e non mi avete vestito, malato e in carcere e non mi avete visitato: via da me, maledetti'»), nell'Inferno. Si può dire: qui gli eletti, lì i dannati, nel mezzo Cristo giudice, del resto è proprio in questo modo (e con questa immagine) che Michelangelo ha affrescato magnificamente la Cappella Sistina. Ma si può altresì dire: è qui e ora che si distinguono eletti-felici e dannati-infelici, è qui e ora, infatti, che si sperimenta la gioia di vivere - ad esempio attraverso la relazione amicale, l'accoglienza dell'altro, l'aprirsi al prossimo - o, al contrario, che si prova la tristezza di vivere - come nella ipotesi dell'inimicizia, del rifiuto dell'altro, della chiusura rispetto al prossimo. Detto altrimenti: con la non-violenza attiva, praticata qui e ora, si vive meglio, e dunque salvezza o condanna sono conseguenze (premi o castighi) logiche e pratiche delle nostre scelte e delle nostre azioni.

2.1. Le cose non sono affatto semplici. Meglio d'ogni altra, l'affermazione aristotelica - quanti hanno accolto uomini d'altra razza, la maggior parte sono caduti in preda alle fazioni - riassume la preoccupazione che ogni età (antica, moderna, contemporanea) ha conosciuto e conosce di fronte all'altro, al diverso, ora assumendo un comportamento difensivo, talaltra uno decisamente ostile. Pur nella varietà degli approcci e delle definizioni, in tutte le età lo sconosciuto in terra altrui vive una situazione scomoda, se non drammatica, a suo carico sembrano rincorrersi senza sosta discriminazione, giudizio critico, sospetto.

Lo straniero con la sua presenza contamina e minaccia uomini e luoghi, è poco familiare ed è colpevole sino a prova contraria. Ambiguo e oscuro, egli porta con sé una propria visione del mondo, una propria cultura, propri costumi. Nell'antichità avrebbe dovuto compiere sacrifici per purificare il luogo dall'ira degli dèi e dall'epidemia causata dal suo arrivo, e sarebbe stato obbligato a fornire una sorta di compenso, nella forma di notizie da terre lontane o 
di doni per l'ospite. Solo grazie a questo compenso-purificazione egli sarebbe stato accolto nella comunità e sarebbe sfuggito a quei tanti pregiudizi che l'arroganza e la superbia causano.

Anche nella nostra società globale, liquida, flagellata dalla paura di perdere il proprio posto nella società, i migranti continuano a essere percepiti come messaggeri di cattive notizie (per dirla con Bertolt Brecht), o come walking dystopias (distopie che camminano, per dirla con Zigmunt Bauman).

Distopie che nuotano, che camminano. Lampedusa, Calais, Ventimiglia, e, ancora, la Macedonia, l'Ungheria, l'Austria, la Libia. E poi i tanti non-luoghi: la stazione di Roma e di Milano, i parchi di Belgrado, ma anche i tanti centri (di primo soccorso e accoglienza, di accoglienza per richiedenti asilo, di identificazione e di espulsione). Un esodo nel senso letterale del termine: scappano gli afgani, gli eritrei, i siriani, e intanto l'Europa cerca disperatamente una voce comune che oscuri la xenofobia, predispone quote migratorie più o meno rigide, prova a ricollocare. Sarà vero, non lo sarà, quanto stimato dall'antropologo Michel Agier ${ }^{17}$ : un miliardo di sfollati nei prossimi quarant'anni, quel che però è senz'altro vero è che accanto a quella di capitali, di beni e di immagini, è ora arrivato il tempo della globalizzazione dell'umanità che irrompe materialmente nelle nostre strade, con tutti i suoi effetti collaterali.

3. Intorno ai processi migratori si sviluppano da sempre due fondamentali interpretazioni ${ }^{18}$ : l'una orientata a prendere sul serio la comunità e l'appartenenza del cittadino - cioè di colui che è titolare dei diritti civili, politici e sociali, e che ritiene di poterne godere con i propri discendenti in modo pieno ed esclusivo - l'altra orientata a prendere sul serio l'individuo e i diritti, compresi i diritti dello straniero e innanzitutto «il diritto di uno straniero che arriva sul territorio di un altro Stato di non essere trattato ostilmente» ${ }^{19}$.

Due interpretazioni diverse: in base alla prima, i cittadini sono liberi di governarsi a vicenda e di rendere l'appartenenza esclusiva, del resto il consorzio dei cittadini ha un carattere proprio e ha il diritto di conservarlo; diversamente, in base alla seconda interpretazione, gli stranieri non possono perdere

17 Aux bords du monde, les réfugiés, Flammarion, Paris, 2012.

18 Greblo, E., Etica dell'immigrazione. Una introduzione, Mimesis, Milano, 2015.

19 Kant, I., Zum ewigen Freiden (1795), trad. it., «Per la pace perpetua. Progetto filosofico. Secondo supplemento (articolo segreto per la pace perpetua)», in ID., La pace, la ragione e la storia, Il Mulino, Bologna, 1985, p. 114. 
le loro caratteristiche e annullare loro stessi, del resto prima o poi la stessa generazione e/o quelle successive riscoprono le loro origini e la loro cultura. Due interpretazioni che rinviano ora all'assimilazionismo e ora al multiculturalismo, vie in fondo impraticabili. Con il primo, si pretende che lo straniero diventi simile: cioè che si adatti e faccia sua la cultura del paese che lo accoglie, abbandonando i propri riferimenti culturali. In breve, si pretende che egli annulli se stesso. Con il secondo, invece, si tende a garantire i diritti culturali delle comunità, dei gruppi e delle minoranze, favorendo la loro promozione nello spazio pubblico. Il consorzio dei cittadini viene così presto smantellato per far spazio ai diversi consorzi di estranei. Nell'uno e nell'altro caso, il problema rimane aperto e la realtà si dibatte tra l'omologazione e la convivenza tempestosa.

I dialoghi fantascientifici di Bruce Ackerman ${ }^{20}$ restano decisamente attuali e di grande rilevanza, nella conversazione tra il passeggero dell'Apollo e il passeggero dell'Explorer, si ritrovano quelle che - di fatto - rappresentano le questioni ultime: «perché pensi di avere i requisiti di cittadino, mentre io non li avrei? [...] se la cittadinanza è il più importante diritto nella teoria liberale, come puoi permettere che questo diritto venga concesso per ragioni che non accetteresti mai su questioni di minore importanza? [...] Dopo tutto la stai usando come ragione per negarmi tutti i diritti!».

3.1. I due poli di un dibattito filosofico sul fenomeno migratorio quanto mai vivace possono essere oggi ben rappresentati da Michael Walzer ${ }^{21}$, che privilegia l'autonomia politica rispetto al diritto di libera circolazione e quindi riconosce al potere sovrano la facoltà di definire le regole di attraversamento dei propri confini quale condizione di esistenza della comunità politica stessa, e da Joseph Carens ${ }^{22}$, che considera la libera circolazione un diritto umano fondamentale e che, poiché i diritti morali possono essere ricondotti solamente agli individui uti singuli e non ai gruppi e alle comunità, equipara le contemporanee restrizioni all'immigrazione agli insopportabili criteri di distribuzione in base alla razza e al sesso.

20 Social fustice in The Liberal State (1980), trad. it., La giustizia sociale nello stato liberale, Il Mulino, Bologna, 1984, p. 144.

21 Spheres of Justice: a Defense of Pluralism and Equality (1983), trad. it., Sfere di giustizia, Feltrinelli, Milano, 1987; What It Means To Be An American: Essays on the American Experience (1992), trad. it., Che cosa significa essere americani, Marsilio, Venezia, 1992.

22 The Ethics of Immigration, Oxford University Press, New York, 2013. 
Tra le due ricostruzioni proposte, una terza via è d'obbligo. E questo perché, tanto la prospettiva morale ideale - non si può negare a ogni essere umano il diritto fondamentale di circolazione, giacché non esiste il diritto di occupare un luogo -, quanto la prospettiva dell'autonomia politica - la democrazia ha bisogno di confini per funzionare, poiché è necessario delimitare la rappresentanza molto semplicemente per sapere chi è responsabile e rispetto a chi - presentano un nucleo essenziale che non può non essere condiviso. La proposta di confini permeabili, porosi - per dirla con Seyla Benhabib ${ }^{23}$ - può costituire un punto di vista significativo. Peraltro, si tratta di un aspetto che è proprio della struttura stessa di ogni ordinamento democratico che, per un verso, fonda la propria legittimità e quella delle sue leggi sulla partecipazione diretta di tutti coloro che sono tenuti a prestarvi obbedienza e, per l'altro, impone vincoli di auto-riflessione, dovuti alla partecipazione nella comunità, in cui si situano le mediazioni tra gli obblighi universali e le necessità di autodeterminazione. Vincoli che, di fatto, si traducono in un processo di perenne riconfigurazione dei confini.

4. La società globale sperimenta nuove forme di interazione giuridica e politica, crea nuovi soggetti che dalla loro capacità di dominare il tempo acquistano potere, pone lo Stato in un contesto di interdipendenza strutturale, cosicché le concezioni moderne di cittadinanza e sovranità devono essere riviste. Diritto e identità rappresentano ormai le due parole chiave tra gli Stati sovrani e la società globale. È sufficiente qui ricordare lo sviluppo del diritto internazionale. Con il riconoscimento di poteri, limiti, diritti e doveri, si è inaugurata una nuova era delle relazioni internazionali: quella della tutela dei diritti dell'uomo e, con essa, della sfida al principio della non interferenza negli affari interni di uno Stato. Si badi: una tutela dei diritti umani via via più ampia, non più solo a portata individuale, ma anche collettiva e con contenuto solidaristico. Nelle nostre società, poi, la costruzione dell'identità si sviluppa anche grazie alla globalizzazione dei media e alla comunicazione elettronica. Sia con l'una che con l'altra, il legame tradizionale tra ambiente fisico e situazione sociale cambia: si riconfigurano le identità (individuali e collettive) a partire dall'interconnessione generalizzata di tutti i terminali, interconnessione che mutua

23 The Claims of Culture: Equality and Diversity in the Global Era (2002), trad. it., La rivendicazione dell'identità culturale. Eguaglianza e diversità nell'era globale, Il Mulino, Bologna, 2005. 
dalla metafora dell'elettrone il proprio carattere libero. I nuovi meccanismi di identificazione trascendono i confini nazionali: qualche volta affermano un'appartenenza globale, qualche altra si inscrivono in una cultura nomade e qualche volta ancora si aprono alle formazioni post-moderne monotematiche. In ogni caso, un aspetto essenziale della costruzione dell'odierna identità è che essa si apre simultaneamente al globale e al locale e, se nel globale non vi è differenza tra interno ed esterno, nel locale il qui e ora diventa sempre più particolare e più radicale.

Tutti temi e tutte riletture, che certo agevolano la comprensione dell'espressione cittadinanza, ma che lasciano aperta la querelle tra diritti dell'uomo e diritti del cittadino. Quali soluzioni per l'odierno fenomeno migratorio? Intanto il recupero di ciò che è oggi in crisi: la fiducia, come pure l'attenta comprensione dell'estraneità e la costruzione di un nuovo paradigma fondato sulla solidarietà.

4.1. La crisi di fiducia affligge di norma le società differenziate e complesse, in altre parole le società avanzate ${ }^{24}$. La diffidenza nei confronti delle istituzioni pubbliche e, in particolare, rispetto a chi le guida (manager e finanzieri, scienziati e medici, politici, giornalisti, e così via) è il tratto che più d'ogni altro unisce e accomuna tra loro i cittadini che, non disponendo di informazioni adeguate o di conoscenze dirette, non riescono a verificare la credibilità delle affermazioni scientifiche, politiche, economiche, per la conoscenza del mondo socialmente rilevante. Assai spesso, anzi, molte affermazioni sono considerate con sospetto, poiché sembrano tradire (o tradiscono) secondi inconfessabili fini. Eppure la natura dei rapporti è così intricata da poter dire che non ci si fida mai, ad esempio, dei media e dei loro resoconti, anche se in realtà per certi versi si dipende proprio da questi. In altri termini, pur diffidandone e provando ad essere cauti nelle nostre adesioni, la nostra conoscenza degli eventi e degli accadimenti viene acquisita sia direttamente che indirettamente, e quindi anche attraverso la lettura e la visione (di quotidiani, di programmi, di siti). Ne diffidiamo e, tuttavia, non possiamo fare a meno di guardare la televisione!

Il fatto è che la mancanza di fiducia ha effetti negativi sulla società e sulla democrazia. È possibile ripristinarla? L'affronta da par suo Onora

24 Luhmann, N., Vertrauen: Ein Mechanismus der Reduktion sozialer Komplexität, Enke, Stuttgart, 1968. 
O'Neill ${ }^{25}$, evidenziando i luoghi comuni ricorrenti, affrontando questioni concrete: trasparenza dell'informazione, cultura dei doveri, burocratizzazione dei controlli, libertà di stampa, e sollecitando una rinascita delle relazioni fiduciarie, risorsa immateriale a disposizione di tutti, bene pubblico indispensabile alla vita economica, sociale e politica.

Innanzitutto, ciò che non consente di spiegare in senso proprio la(e) relazione(i) di fiducia, dal momento che svolge(ono) un ruolo fondamentale nelle nostre vite ed è(sono) condizione(i) di possibilità o di successo di molte delle azioni che pianifichiamo, va rielaborato e corretto. Ed ecco l'avvertenza: la ricostruzione filosofica muove (deve muovere) da inizi e principi semplici per raggiungere conclusioni complesse e importanti. Del resto, è evidente che a nulla servirebbe la possibile inversione: partire da assunzioni eccessive e straordinarie per concludere con giustificazioni incerte e limitate. Secondo O'Neill, diventa necessario muovere dall'autonomia kantiana e, in particolare, dalla sua visione umile dei poteri della ragione, si badi: umile ma non scettica. Kant ha, infatti, ben chiari i limiti della ragione umana ed è per questo che si occupa dell'autorità legittima della ragione; finiti e limitati, gli esseri umani sono inclini all'errore, all'arroganza, alla presunzione, e proprio per questo hanno bisogno di ricorrere al ragionamento, di sottoporre credenze e idee alla valutazione razionale. Certo, la ragione umana può errare e le verità di ragione non riescono sempre ad essere provate senza ombra di dubbio; tuttavia, la distinzione tra ciò che è vero e falso, ciò che è giusto e ingiusto, permane e richiama criteri di giustificazione che abbiano carattere pubblico, cioè possono essere comunicati (agli) e scambiati con gli altri. Ragioni, quindi, che anche gli altri possano condividere e concezione non individualistica dell'autonomia, concezione perciò di tipo giuridico. Sottolinea O'Neill'26 l'autonomia kantiana si riferisce ad agenti che agiscono in modo libero e sulla base di principi condivisi universalmente. Diversa dalla libertà (che può essere usata anche per azioni malvagie e che in ogni caso non è sufficiente a mostrare la giustificazione morale di un'azione, se non nell'ipotesi in cui sia moralmente obbligatoria) e diversa dalla semplice autonomia individuale (anch'essa insufficiente a determinare l'accettabilità morale di quanto richiesto o invece proibito), l'autono-

25 O’NeILl, O., A Question of Trust. Traduzione italiana Una questione di fiducia, Vita e Pensiero, Milano, 2003.

26 Constructing Authorities: Reason, Politics and Interpretation in Kant's Philosophy. Cambridge University Press, New York, 2015. 
mia kantiana richiede che i principi, perché possano dirsi universali, debbano poter essere seguiti da tutti, giacché la loro adozione consente di conservare, anziché di distruggere, di rafforzare, piuttosto che di indebolire, le capacità che le persone hanno di agire secondo quegli stessi principi.

Di qui la fiducia, non come sentimento, bensì come azione comunicativa con la quale si attribuisce credibilità agli altri, e la si attribuisce proprio in quanto criteri, procedure e vincoli, costituiscono la base di riferimento per discriminare tra coloro che meritano la fiducia, o al contrario non la meritano. Una base di riferimento che non sempre evita tradimenti e manipolazioni. $\mathrm{Nel}$ dibattito su Brexit, sostiene O'Neill in un'intervista alla BBC, sono mancati resoconti onesti e affidabili da entrambi le parti, pregiudicando il dibattito, disinformando l'opinione pubblica, indebolendo la fiducia reciproca che è condizione della cooperazione sociale ${ }^{27}$.

4.2. Senza la fiducia, il nuovo articolarsi dell'estraneità (lo straniero esterno - il pericolo, la paura - e lo straniero interno - e cioè l'immigrato, ma anche il lavoratore precario, il disoccupato, il ceto medio, come pure il greco, l'italiano) resta impigliato nelle maglie di un'Europa tecnocratica e nichilista, non consapevole dei suoi tesori: la diversità delle lingue, la conquista del dubbio, il successo di valori, quali la libertà, la democrazia, la cittadinanza, in breve i diritti dell'uomo. Per far sì che questi tesori non siano irrimediabilmente persi occorre una nuova pratica politica. Anche per il chi siamo, come del resto per il chi sono, la migliore risposta è una inquietudine interrogante, possibile solo a partire da quella realtà europea identitaria, che accoglie con sé e raccoglie in sé lo straniero, e da quel vigore storico rappresentato dalla memoria culturale del nostro continente. Un nuovo umanesimo - per dirla con Julia Kristeva ${ }^{28}$ in grado di fronteggiare i due mostri che minacciano il pianeta globalizzato, ovvero la politica chiusa tra economia e finanza e l'autodistruzione ecologica. $\mathrm{E}$ in un contesto siffatto, l'esperienza culturale e la risposta che si darà alla questione identitaria hanno un'importanza decisiva.

Una nuova pratica politica richiede però che si ragioni seriamente sull'essere stranieri e sull'essere percepiti come stranieri, intanto perché ciascuno di

27 After the Vote, in The Brexit Collection, <http://www.bbc.co.uk/programmes/articles/2CK7XGqZlqPDjJMJ8dbXSGd/a-point-of-view-after-the-vote> (visitato il 24 gennaio 2018).

28 Etrangers à nous-mêmes (1988), trad. it., Stranieri a noi stessi. L'Europa, l'altro, l'identità, Donzelli, Roma, 2014. 
noi è «straniero a se stesso», è unico ed è molteplice, è singolare ed è plurale: lo straniero è scoperta dell'altro, e ancor prima di qualsivoglia considerazione quest'altro è il suo corpo con i suoi segni distintivi naturali e/o culturali, e poi anche perché nel riconoscersi tutti stranieri si eviti di cadere nell'a-polidia, ovvero in un processo de-socializzante, senza più cittadinanza e solidarietà ${ }^{29}$.

Si badi: senza la fiducia e senza la solidarietà non resta altro che una società disgregata e totalitaria, nella quale valori e diritti, quali: cittadinanza, libertà, democrazia, reciprocità, sono semplicemente dei valori e dei diritti tra gli altri; valori e diritti di un gruppo etnico che convive con altri gruppi etnici che spesso professano e reclamano valori e diritti antitetici. Fianco a fianco con gli altri e le altre, si è così immersi in una realtà in cui il femminismo - magari radicale - convive con orribili forme di oppressione della donna e nella quale il laicismo - anche quello aggressivo - si autocensura pur di fronte a forme minacciose di fanatismo religioso, e dove la libertà, che certe volte tende a sconfinare nel libertinismo, convive, come se niente fosse, con violazioni evidenti dei diritti umani.

E intanto che gli scontri etnici risorgono e aumentano, il diritto internazionale, il diritto della navigazione, il diritto costituzionale, il cosiddetto diritto penale interculturale, diventano oggi teatro di discussioni tanto aspre quanto ardue circa lo spazio da riconoscere alle aspettative normative generate dalle diverse culture e dalle differenti religioni. Si pensi alla problematica medicalizzazione di alcune pratiche, alle discriminazioni tra i sessi tollerate - se non persino imposte - da determinate tradizioni, alla difficile composizione di concezioni dell'istituto familiare molto lontane tra loro, o, ancora, al ruolo pubblico delle religioni e delle loro concezioni istituzionali nelle società occidentali, che dall'illuminismo in avanti hanno progressivamente limitato la presenza e la visibilità sociale del fenomeno religioso fino a teorizzarne la privatizzazione.

4.3. Nelle società attuali siamo diventati membri di minoranze che si costituiscono di volta in volta a seconda dei problemi all'ordine del giorno. Come realizzare allora una convivenza pacifica in società ormai abitate solo da stranieri e soltanto da minoranze?

29 Todorov, T., La Conquête de l'Amérique: La Question de l'autre (1982), trad. it., La conquista dell'America. Il problema dell'altro, Einaudi, Torino, 1997; Amato Mangiameli, A.C., Sfide di teoria giuridica, Cedam, Padova, 2010. 
L'espressione solidarietà tra estranei di Jürgen Habermas ${ }^{30}$ è qui particolarmente utile. Quando gli individui si incontrano e vivono all'interno degli stessi spazi, non avendo alcunché in comune, non condividendo eguali idee e opinioni, ciò che può consentire una convivenza pacifica è il diritto quale medium dell'integrazione sociale. Una simile capacità integrativa non deve essere ricondotta semplicemente alle proprietà formali del diritto - e quindi al suo articolarsi in termini di diritti soggettivi - oppure al suo essere prodotto da un legislatore e al suo carattere coercitivo, ma deve essere ricondotta soprattutto al fatto che gli individui che si incontrano e che vivono all'interno degli stessi spazi, pur non avendo alcunché in comune, possono insieme - e allo stesso tempo - accettare le norme statuite come norme legittime che meritano un riconoscimento intersoggettivo. Sotto questo profilo, il diritto si confa perfettamente alla coscienza morale post-tradizionale, secondo la quale i cittadini sono disposti ad obbedire ai comandi sulla base delle loro buone ragioni. E le ragioni diminuiscono comunque la probabilità che il diritto statuito venga usato arbitrariamente o che il senso delle norme e delle procedure rimanga fondamentalmente oscuro.

Se così, la prestazione integrativa principale svolta dal diritto è quella di rafforzare persuasivamente quella debole morale post-tradizionale che si ritrova radicata nella coscienza della persona, coscienza che - a sua volta - quasi in un rapporto di reciprocità, verrebbe mobilitata in occasione della legittimazione del diritto.

«Questi estranei noi li sussumiamo comunque nella categoria dell'altro, sia che essi appartengano alla nostra stessa nazione (e siano nostri concittadini) sia che non vi appartengano. Le obbligazioni speciali che ci legano agli 'altri' non derivano in primo luogo dall'appartenenza a una certa comunità concreta. Esse derivano piuttosto dall'astratto coordinamento d'azione operato da istituzioni giuridiche, nel momento in cui queste istituzioni ascrivono determinate obbligazioni a certi gruppi di persone o a certi soggetti, al fine di esplicitare sul piano sociale e di contenuto (nonché di rendere vincolanti) obblighi positivi che altrimenti resterebbero indeterminati ${ }^{31}$.

La solidarietà tra estranei e la morale post-tradizionale debole potrebbero garantire la convivenza pacifica anche con la nuova immigrazione, sem-

30 Die Einbeziehung des Anderen. Studien zur politischen Theorie (1988), trad. it., L'inclusione dell'Altro. Studi di teoria politica, Feltrinelli, Milano, 1996.

31 Habermas, J., Morale Diritto Politica, Einaudi, Torino, 1992, p. 131. 
preché si sviluppino politiche adeguate, capaci di offrire servizi, di reprimere comportamenti illegali e, in generale, di predisporre filtri di qualità e di rigore. Resta però il fatto che, nel caso del conflitto di valori tra culture diverse, tutto dipende dalle tradizioni e dalle appartenenze degli immigrati: alcuni riescono a inserirsi subito e con successo nelle società europeo-occidentali, altri si inseriscono con ritardo e superando una serie di difficoltà, altri ancora restano sempre sulla soglia.

5. L'odierna giustificazione dell'obbligatorietà normativa deve essere capace di integrare creativamente quelle dimensioni del moderno che sono da considerare acquisizioni irrinunciabili e quelle altre che pur importanti sono state sacrificate sull'altare della modernità. Questo sforzo critico diventa tanto più prezioso se si considerano le nuove forme del dominio, che trascendono i confini nazionali, assumono un carattere opaco e diventano pure forme di manipolazione di individui e collettività, rispetto alle quali è perciò importante la riproposizione di forme istituzionali (lo Stato, l'Unione europea, la Comunità Internazionale) e di relazioni giuridiche (tra cittadini, tra cittadini europei, tra stranieri) all'insegna della pacifica convivenza, dell'integrazione e della solidarietà.

Si tratta di riguadagnare l'essenza del diritto, e con essa il senso dei trattati e il significato delle carte fondamentali, soprattutto in un momento di grande cambiamento, in un momento in cui i processi di internazionalizzazione economica determinano un aumento abnorme del capitale finanziario a discapito del suo utilizzo proprio nel ciclo del lavoro e della solidarietà.

Avverso questo aumento, l'idea moderna della relazione diritto-diritti-solidarietà, con i suoi sviluppi, può rivelarsi un'importante chiave di lettura. La solidarietà come elemento costituzionale dell'ordinamento statale, europeo e internazionale si è potuta affermare grazie allo spostamento concettuale dalla proprietà al lavoro ${ }^{32}$. Non più i beni in mio potere determinano la mia indipendenza, bensì le mie capacità lavorative, sviluppate in un contesto in cui l'educazione, lo studio e la ricerca diventano essenziali per il modello di organizzazione pubblica e sociale. Anche il principio di universalità di alcuni diritti

32 La grande proprietà diventa irrilevante per l'organizzazione politica dello Stato, ma può ancora svolgere un ruolo se rientra nel ciclo del lavoro, se cioè si atteggia a volano del sistema solidaristico. Come affermava la Costituzione di Weimar, «la proprietà obbliga». 
(salute, assistenza, educazione, ecc.) è legato all'affermazione che chi lavora sopporta l'onere dei costi dei diritti di partecipazione, dei diritti economici e dei diritti sociali. Lavoro e solidarietà compongono così un sistema di diritti di carattere circolare.

Avverso l'aumento abnorme di capitale finanziario, che tende a mettere in discussione proprio i diritti solidali e il lavoro, riducendo quest'ultimo al rango di una qualsiasi merce, l'idea contemporanea della relazione diritto-diritti-solidarietà deve muovere dalla rilettura di bene (beni) e, in particolar modo, dall'affermazione - rispetto ai beni esclusivi, dei cd. beni inclusivi - che essi proprio perché tali superano i limiti della politica e dell'economia tradizionali, mettono al centro la relazione, prediligono la spiritualità sulla materialità.

«La loro logica è la gratuità: liberamente vengono creati, liberamente circolano e vengono fruiti. La divisione è la logica dei beni esclusivi, dove il riconoscimento del diverso è tanto più difficile quanto più si entra in concorrenza, la partecipazione quella dei beni inclusivi. Per aumentare le probabilità della pace sono necessari tanto una giusta ripartizione dei beni esclusivi, quanto un incremento della sfera dei beni inclusivi» ${ }^{33}$.

E del resto, se rettamente inteso, il diritto - con la sua categoria essenziale, la giustizia - appartiene all'ordine dei beni inclusivi.

33 Possenti, V., Il nuovo principio persona, Armando, Roma, 2013, p. 251. 